\title{
Improvements in Ultrasonic Inspection of Resistance Spot welds
}

\author{
Joe Buckley ${ }^{1}$, Ramon Servent ${ }^{2}$ \\ 1- Sonatest Ltd, Milton Keynes, ENGLAND \\ 2- Atlantis NDE, Madrid, SPAIN
}

Joeb@sonatest.com

\begin{abstract}
Resistance welding is an electrothermic process commonly used in Industry for joining sheet metal, particularly steel, in applications such as automobile bodies and chassis assemblies. The method is suitable for partial or total automation, and is very reliable. However, as with any process, problems can occur and inspection is therefore necessary. Although ultrasonic inspection of spot welds has been used for many years, the technology still has problems with production rate and reliability, and is very operator dependent. This paper will look at the analysis of ultrasonic signals from weld inspection, and present some improvements in both probe technology and automatic analysis. Together these result in significant improvements in inspection reliability, and allow the inspection to be fully automated.
\end{abstract}

Keywords: Automotive, Resistance Welding, Sheet Metal.

\section{Introduction}

Resistance spot welding was developed around 120 years ago, and has been widely used as a fast, low cost and reliable technique for joining sheet metal. Typically two sheets of metal are held together between copper electrodes; pressure and a large pulse of current is applied, and heat is produced, primarily at the interface between the two sheets of metal, resulting in melting and the formation of a strong weld. Then the current is turned off, after a short cooling period the pressure is removed, and the cycle continues at the next point. The total time to produce an individual weld can be of the order of two or three seconds or less.

Unfortunately, although the process is in principle straightforward, there are many things that can go wrong:

- The process parameters of time, pressure and current must be correctly controlled. Given modern, well maintained equipment and a correctly designed process this should seldom be an issue, but errors can occur.

- Contamination, which could include oil or oxides as well as material problems such as plating thickness variations.

- Electrode wear/degradation. This is usually the biggest issue. There is an obvious trade off here - it is expensive to replace the electrodes too early. But as they wear problems may start to occur, and issues such as contamination, hollowing, coating material pick-up, poorly controlled pressure or pulse timing (contact 'bounce' while current is applied may cause arcing) and surface plating variations can all lead to faster wear than expected.

So a variety of weld conditions or defects can be identified as shown in Table 1

Any means of evaluating welds must, as a minimum, be able to reliably confirm that a weld is 'good'; if we also wish to use inspection as a tool to resolve production problems we must be able to reliably confirm the type 
of problem present. Traditionally a 'chisel test' has been used, but this is not reliably non destructive and, unless a part is broken apart completely, it will not give us much information on the defect type.

Table 1: Resistance weld Identified conditions

\begin{tabular}{|c|c|c|}
\hline Condition & Image & \\
\hline Good Weld & & \\
\hline $\begin{array}{l}\text { 'Burnt' Weld }- \text { Melt } \\
\text { zone extends to surface }\end{array}$ & & $\begin{array}{l}\text { High current } \\
\text { or high initial } \\
\text { resistance, too } \\
\text { long current } \\
\text { time, }\end{array}$ \\
\hline $\begin{array}{l}\text { 'Stick' weld } \\
\text { Very small melt zone. } \\
\text { Maybe just coating bonded } \\
\text { together }\end{array}$ & & $\begin{array}{l}\text { Not enough or } \\
\text { poorly controlled } \\
\text { current, could be } \\
\text { too low } \\
\text { resistance due to } \\
\text { coating, excess } \\
\text { pressure }\end{array}$ \\
\hline $\begin{array}{l}\text { 'Loose' weld } \\
\text { No bond at all }\end{array}$ & & As above \\
\hline $\begin{array}{l}\text { Undersize weld } \\
\text { Melt has not spread far } \\
\text { enough }\end{array}$ & & $\begin{array}{lr}\quad \text { Not } & \text { enough } \\
\text { energy, } & \text { short } \\
\text { pulse } & \text { or } \\
\text { electrode } & \\
\text { damage, } \\
\text { contamination }\end{array}$ \\
\hline $\begin{array}{l}\text { Defects } \\
\text { Inclusions, cracks or } \\
\text { porosity in weld. }\end{array}$ & & $\begin{array}{l}\quad \text { Too much } \\
\text { current, } \\
\text { Contamination, } \\
\text { electrode } \\
\text { damage, low } \\
\text { pressure }\end{array}$ \\
\hline
\end{tabular}

Ultrasonic methods of spot weld evaluation have been used in the automotive industry for around 20 years and can be extremely effective. However they have required a high degree of operator skill and, especially when this is not available, have a relatively poor confidence rate.

The quality of a spot weld is determined according to particular engineering criteria by the correlation between strength and the different parameters that can be measured in the ultrasonic test: i.e. thickness, attenuation rate, presence of internal flaws and nugget diameter.

This highlights the need for a quantitative, proper, ultrasonic evaluation of the spot weld, and for an equally quantitative definition of the appropriate rejection criteria (thresholds) for each spot weld. This implies that a 
correct setup of the evaluation system shall comprise a proper calibration with suitable reference standards or samples.

To optimise the reliability of the ultrasonic evaluation of spot welds we must firstly improve performance of the ultrasonic acquisition, signal selection and signal evaluation itself, this is applicable to both manual and automatic inspection.

Secondly we need to overcome the operator related reliability factors by the implementation of an automatic inspection process at the same time this can improve testing efficiency, increase the possible scope of the inspection, and will thus allow 'real in-line' spot weld testing, without comprising the performance of the production line as a whole

This paper describes work to define a more general approach to weld classification which can be automated and give a high level of reliability by improving the following aspects of the inspection system

- $\quad$ Practical ultrasonic issues - Probe positioning and coupling

- Capture of a 'good' signal

- Evaluation of that signal

Obviously all are interrelated and must all be optimised for a good overall performance to be obtained.

\section{Signal Optimisation}

Consistent probe coupling is improved by using a patented dry 'rubber' coupling material shown in Figure 1 which is less affected by wear and surface conditions, and which is in particular robust enough to be positioned by an industrial robotic system.

Proper probe positioning requires that the probe is reliably centred on

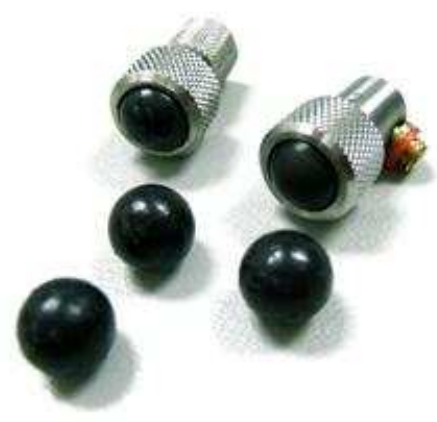

Figure 1: Ultrasonic probes and tips for spot weld inspection the weld spot, normal to the weld surface (which may not be exactly aligned to the material) and has a consistent coupling quality. In practice this is extremely difficult to achieve and maintain, especially when applied manually, so the software must be capable of 'grabbing' a good signal as it appears so that it can then be analysed .

Ultrasonic signals obtained from inspection of spot welds have been examined in detail elsewhere, [1,2] so will only be summarised:

Figure 2 shows typical echo patterns, comprising:

1. A decaying pattern of multiple "primary echoes" from the through thickness of the weld.

2. A 'Napoleons hat' pattern of echoes caused by the weld nugget being smaller than the sound field

3. A decaying pattern of intermediate echoes if there are any defects within

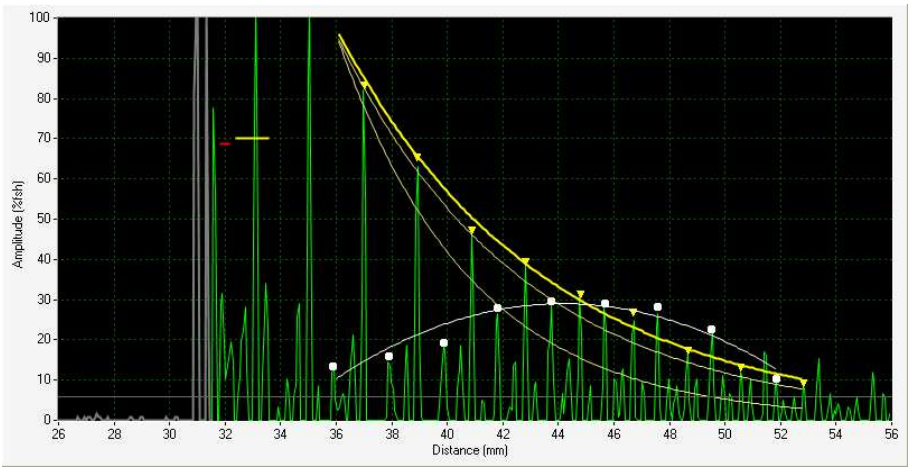

Figure 2: Primary and secondary echo patterns the weld nugget. 
So reliability in ultrasonic (manual) spot weld inspection is affected by the correct positioning of the probe onto the centre of the spot weld, its relative orientation to the surface of the spot weld, the quality of the coupling, (which is also related to the pressure on the probe and the spot weld surface condition) and the accurate selection of the signal to evaluate among the number of those obtained during the test.

The negative impact of these factors can be minimized by:

- the larger number of signal captures with real time evaluation

- a careful signal selection and evaluation capability of the test system

- a much better (even) coupling which can be achieved by using dry coupling rubber delays instead of the conventional, wet coupling, bubble probes.

Following a very fast signal acquisition system the ultrasonic signal selection and evaluation criteria perform automatically, based on consistent principles and independent of the particular coupling conditions. This both reduces the dependence of the reliability of a manual inspection on the operator's skills and attention paid to the test and also makes reliable automation of the spot weld inspection feasible.

Additionally, signal evaluation algorithms can be defined though performing the suitable setup adjustments to solve the specific conditions, so as to test spot welds of different materials, including Zinc coated steels, Ultra High Strength Steels, Aluminium, and configurations with two and three sheets .

Dry coupling with solid (rubber) delays, being a significant advantage for manual testing, and is the only way to reliably automate, the spot weld inspection by avoiding the need of a time consuming membrane replacement of the conventional bubble probes every time that it bursts.

In assessing the 'quality' of a signal for evaluation it is useful to consider a number of factors, but the most important are thickness as the distance in between backwall echoes, consistent rate of amplitude decay of the primary echoes (attenuation rate) and relative amplitudes and decay rate of intermediate echoes. 
The software used by AtlantisNDE (Hercules and Cyclops systems) evaluates received signals at approximately $1 \mathrm{kHz}$ for a maximum of a few seconds, selecting the best received, or the first which meets
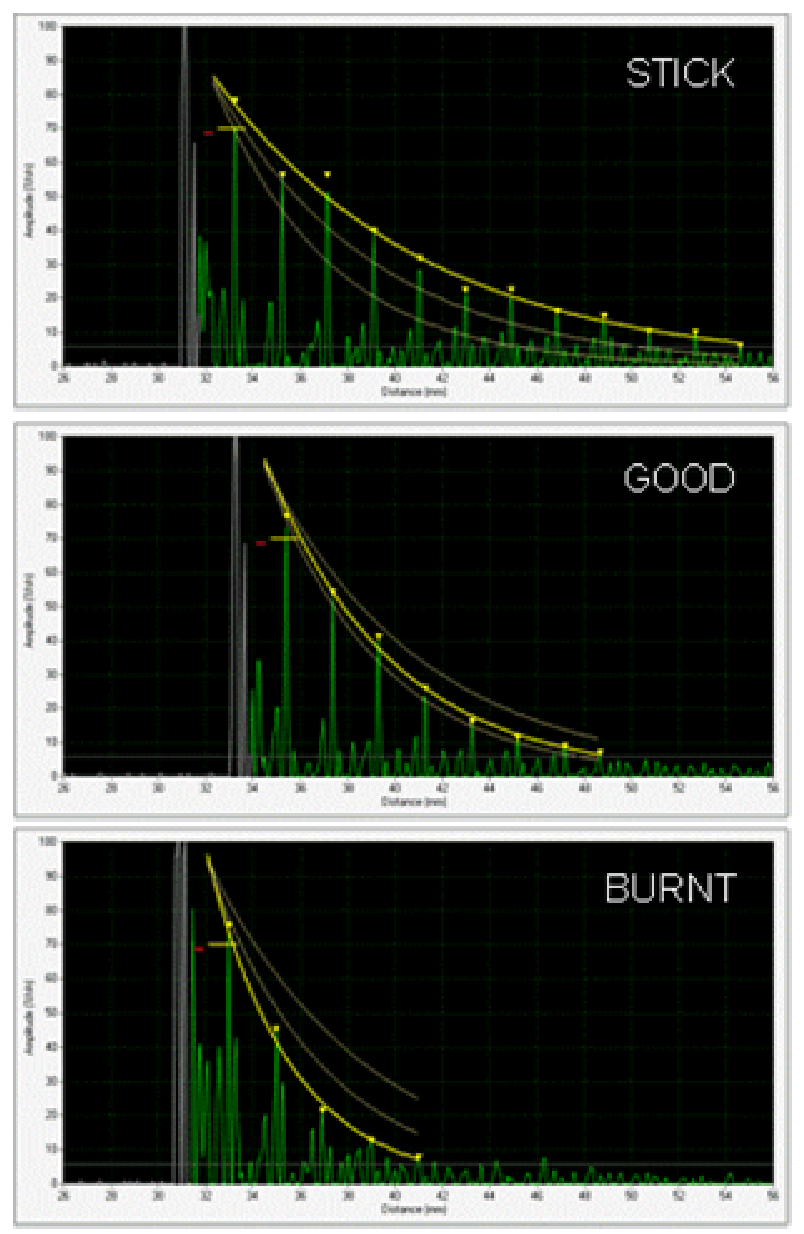

Figure 3: Impact of differing melt extents on primary echo pattern

preset criteria.

\section{Weld Evaluation criteria}

\section{Thickness}

The thickness of the weld can be measured by the average time spacing between 'primary' echoes, divided by the velocity of sound in the metal.

Normally the thickness of a weld will be less than that of the total thickness of the parent metal. The weld process applies pressure to molten metal, so some thinning is to be expected. If the measured thickness is close to $100 \%$ of the total sheet thickness it is likely that little or no melting has occurred, so the weld is likely to be a 'stick' weld or possibly just bonding of the plating. Conversely if the weld is too thin there is likely to be inadequate strength. This allows us to define an acceptable range of measured thickness for the weld. Of course the actual values chosen must be obtained for the particular situation, but we might for example, designate 70$90 \%$ of parent metal thickness as the acceptable range

The thickness measurement will also show a 'loose weld' where the measured thickness is approximately the same as the top sheet of metal.

\section{Back Wall Echoes' Attenuation rate}


Within the weld process very rapid melting and cooling occurs. This results in the material which forms the weld nugget having a relatively coarse crystalline structure which will result in a much higher attenuation rate than unmelted sheet metal. Thus where the nugget is minimal, i.e. a 'stick' weld, the attenuation will be less, and in a 'burnt' weld, when the weld nugget extends throughout the material, the attenuation will be higher than normal. These give very clear changes to the echo pattern, as shown in Fehler! Verweisquelle konnte nicht gefunden werden.

We can therefore determine attenuation rate acceptance thresholds. In a particular weld type we might define example criteria as shown in Table 2

Table 2: Typical attenuation rates

\begin{tabular}{|l|l|}
\hline Attenuation rate & Assessment \\
\hline$<100 \mathrm{~dB} / \mathrm{m}$ & Stick weld \\
\hline $100-400 \mathrm{~dB} / \mathrm{m}$ & Good weld \\
\hline$>400 \mathrm{~dB} / \mathrm{m}$ & Burnt weld \\
\hline
\end{tabular}

It will be noted that we already have two semi-independent factors to improve the confidence of our assessments; both are, at least to some extent, sensitivity independent, meaning that coupling quality, the primary problem in ensuring reliable readings, has a reduced impact.

\section{Intermediate echoes}

Echoes appearing between the primary echo sequence are obviously due to reflectors at an intermediate distance i.e. at the boundary between the metal sheets. As already noted they fall into two general categories:

- On-axis defects such as pores and cracks. The amplitude of these will decay quite rapidly with time. They can be easily identified by looking over the first few microseconds of the display and a 'Flaw' condition identified.

- Off-axis Signals due to the weld nugget not completely filling the sound beam. These will initially increase in amplitude with time, as the beam spread includes a greater fraction of the un-welded sheet on multiple reflections. ;

Under some circumstances, the diameter of the nugget (welded volume) does not reach the nominal diameter of the spot weld and a circular ring around the centre of the weld remains unwelded. An undersized weld thus ultrasonically features a sequence of backwall echoes with attenuation rates appropriate for stick or even good welds and an additional sequence of decaying intermediate echoes at the position of the sheet's interface. The quantitative evaluation of that intermediate echo sequence correlates to the actual nugget diameter which, typically, is required to be larger than $f \sqrt{ } t$, where $f$ is a factor (3.5 or 4) and $t$ the minimum sheet thickness in the stack.

The relative amplitude of the secondary echoes compared to the primary echo locus as shown in Figure 4, correlates well to weld nugget size (Figure 5) We can therefore give s a reliable estimate of the nugget diameter, and compensate for this in the analysis algorithm. In turn, this allows the use of probes somewhat larger than the weld nugget, giving greater flexibility, since the probe need not be uniquely selected for a particular weld type. Use of an 'oversize' probe also provides better tolerance of off-centre probe alignment since, provided the sound beam covers the weld, the positioning is not too critical. 


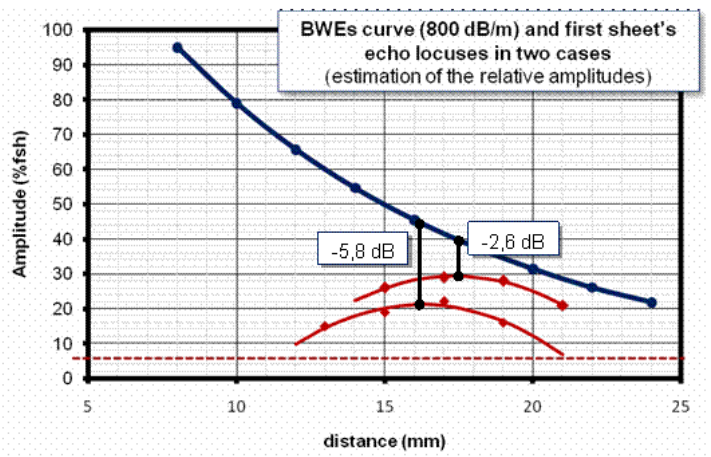

Figure 4: Measurement of gap between primary and secondary echo curves

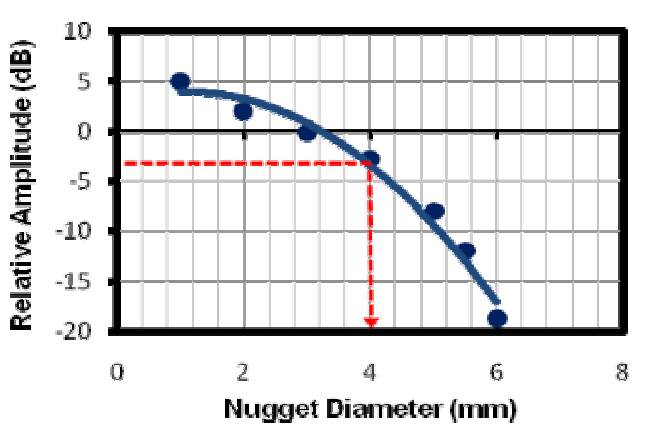

Figure 5: Typical curve of nugget size vs. amplitude

In $\mathrm{Zn}$ coated steels, a weld can be produced in a ring around the diameter of the spot weld whereas the centre of it remains just 'stick' (Zn coating bonding) allowing a partial transmission/reflection of the ultrasonic beam through it. The suitable ultrasonic signal pattern shows then a similar sequence of backwall echoes, with a series of relevant intermediate echoes at the position of the sheet's interface with increasing amplitudes up to a distance and then decreasing (Napoleon's hat pattern).

So by combining these different aspects of the received echo we have an algorithm that:

- Can identify each of the conditions in Table 1.

- Is tolerant of a variation in nugget size with a single probe, so can normally be used to cover the welds in a structure using a single probe / tip combination, minimising the need for manual automation.

- Works primarily in terms of numeric values such as material velocity, thickness and attenuation rate, so can quickly be set to evaluate different weld configurations, and even different metals, such as Aluminium or high strength steels, both of which have caused problems with equipment based on pure 'pattern recognition' algorithms.

\section{Practical Implementation.}

Atlantis NDE have produced three commercially available products based on this ultrasonic technology and analysis algorithm.

HERCULES, (Figure 6, Figure 7) is a system implemented on a laptop PC with an externally mounted ultrasonic card in a PCI expansion chassis $\mathrm{s}$ shown in. This is intended for manual and developmental use

HERCULES with Control plan, This is an enhanced version of the Hercules software, incorporating both weld configuration and results databases: The system is programmed with the weld location (normally a number of annotated digital photographs), and the configuration (number of layers, layer thickness, analysis

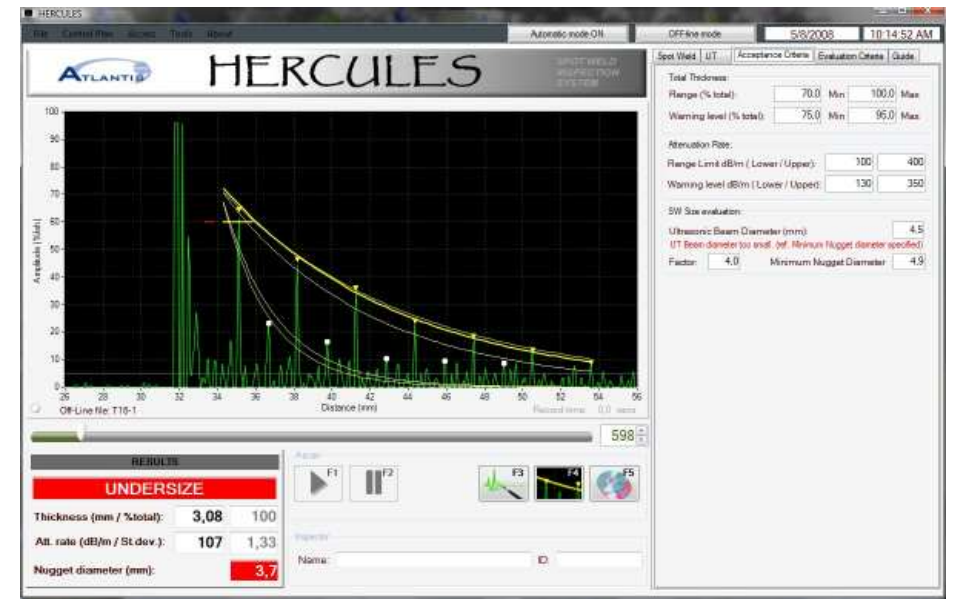

Figure 6:Hercules software user interface 
parameters) of each weld in a part, In use the instrument is automatically reconfigured for the next weld after every reading is taken, giving a high production and low error rate. The operating screen (Figure 8) guides the operator through the sequence.

CYCLOPS [3] is a fully automated production system using an industrial robot, a general view of the system is shown in Figure 9 . A machine vision system (Figure 10) is used to identify the precise location of the weld spot. The robot then places the probe centrally on the weld and optimizes the orientation to obtain the best signal, which is then recorded and analyzed.

The systems can achieve high production rates, up to 1500 welds /shift for the Hercules with Control plan, and approximately 3000 welds/shift with Cyclops

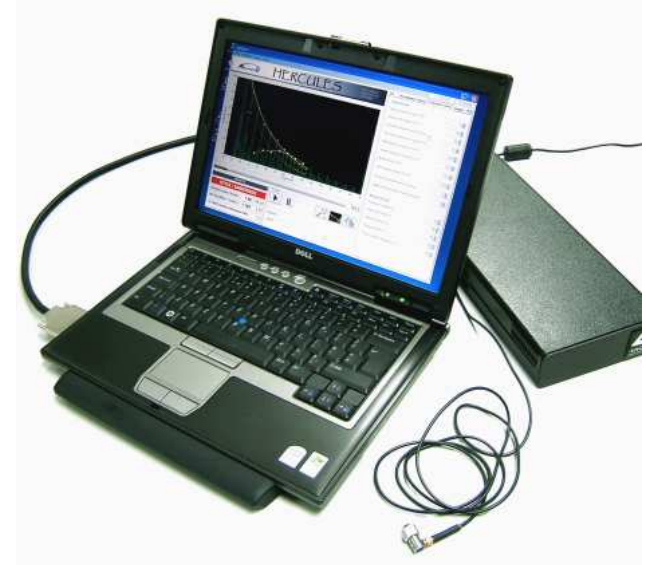

Figure 7: Hercules System

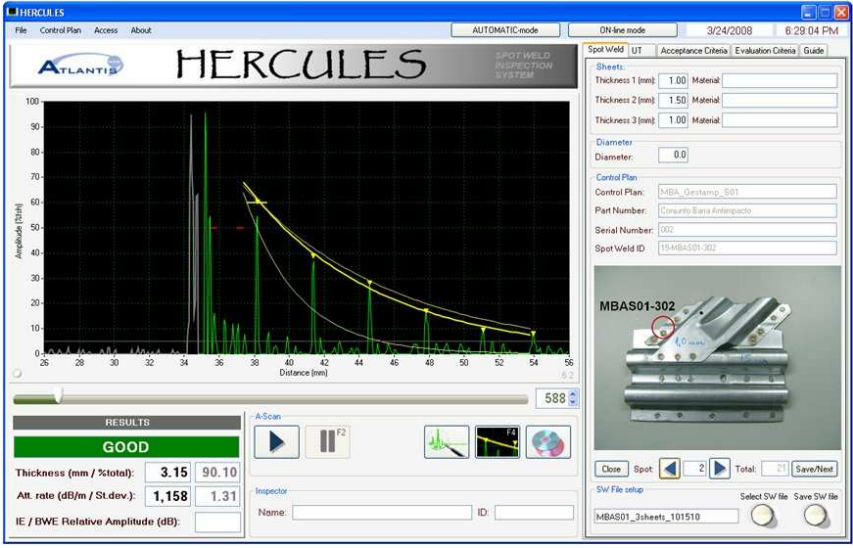

Figure 8: Hercules Control Plan interface

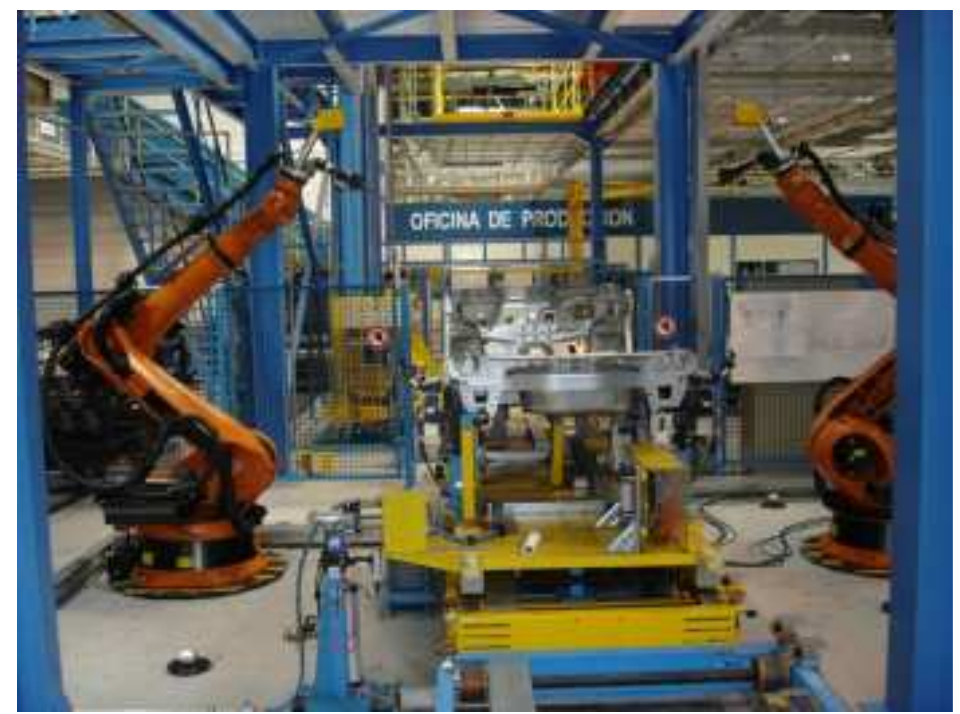

Figure 9: General view of a production CYCLOPS system using dual robotic test heads

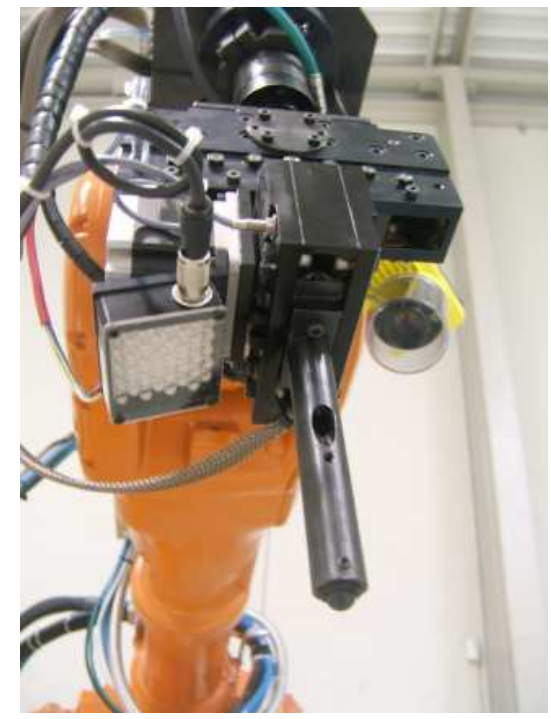

Figure 10: CYCLOPS test head showing machine vision system and probe manipulator

\section{Industrial Experience}

Initial development of the system has been carried out in cooperation with the Ford Motor Company plant in Valencia, Spain. Eight automated systems are currently in use. The first was installed over two years ago. The 
systems are currently operating on a three shift system, and have now tested hundreds of thousands of welds. Correlation to destructive tests has been rated "extremely good", especially on Zinc coated steels.

Nugget diameter measurement has typically been accurate to better than $0.2 \mathrm{~mm}$

Probe delay ball life has been very good typically on machines working near continuously (average around 5-8 measurements per minute) the probe delays (costing a few Euros) are replaced after 20-30 days. This compares extremely well with the 'conventional' liquid filled membrane delay tips, which might last an hour or less in comparable usage.

\section{Conclusions}

The combination of an improved, more reliable ultrasonic coupling technique, Robust and reliable evaluation algorithms, and an optimised automation system has produced a family of inspection approaches which are scalable from low-volume manual operation to the requirements of a modern high speed automated car plant. Currently the system is being implemented in a number of other locations and organisations. The main challenge now is to build on initial favourable results and develop experience with differing situations, in particular the application to Aluminium and other advanced alloys.

\section{References}

[1] "Ultrasonic testing as a means for quality assurance in resistance spot welding". H. Polrolniczak, Moers. Krautkrämer - Sonderdruck . SD 297

[2] "Ultrasonic testing of spot welded joints on coated steel sheets and optimization of welding parameters". R. Kaminski, GE Inspection Technologies Publications, Krautkrämer, Sonderdruck SD296,

[3] Automated Spot Weld Inspection in the Automotive Industry, S. Rivas, R. Servent, Tecnitest Ingenieros SL, Madrid, Spain;, J. Belda, Eines, Valencia, Spain, $16^{\text {th }}$ World Conference in NonDestructive Testing, Montreal, 2004 